\title{
rs744166 Polymorphism of the STAT3 Gene Is Associated with Risk of Gastric Cancer in a Chinese Population
}

\author{
Kexin Yuan, ${ }^{1}$ Huimin Liu, ${ }^{2}$ Lina Huang, ${ }^{1}$ Xiyun Ren, ${ }^{1}$ Jingjing Liu, ${ }^{1}$ \\ Xiaoqun Dong, ${ }^{3}$ Wenjing Tian, ${ }^{1}$ and Yunhe Jia ${ }^{4}$ \\ ${ }^{1}$ Department of Epidemiology, College of Public Health, Harbin Medical University, 197 Xuefu Road, Harbin, \\ Heilongjiang 150081, China \\ ${ }^{2}$ Department of Neurology, The Forth Affiliated Hospital, Harbin Medical University, Harbin, Heilongjiang 150001, China \\ ${ }^{3}$ Department of Biomedical and Pharmaceutical Sciences, College of Pharmacy, The University of Rhode Island, Pharmacy Building, \\ 7 Greenhouse Road, Kingston, RI 02881, USA \\ ${ }^{4}$ Department of Colorectal Cancer Surgery, The Third Affiliated Hospital, Harbin Medical University, 149 Xuefu Road, Harbin, \\ Heilongjiang 150081, China
}

Correspondence should be addressed to Wenjing Tian; twj8267@sina.com and Yunhe Jia; 13945676987@126.com

Received 11 February 2014; Accepted 1 April 2014; Published 23 April 2014

Academic Editor: Gulam Waris

Copyright (C) 2014 Kexin Yuan et al. This is an open access article distributed under the Creative Commons Attribution License, which permits unrestricted use, distribution, and reproduction in any medium, provided the original work is properly cited.

\begin{abstract}
The aim of this study was to explore the association between polymorphisms in signal transducer and activator of transcription protein 3 (STAT3) and the risk of gastric cancer. In the present study, a case-control study was conducted in which rs 2293152 and rs744166 polymorphisms in STAT3 were analyzed in 209 Chinese patients with gastric cancer and 294 cancer-free controls. The genotypes were determined by polymerase chain reaction restriction fragment length polymorphism method. For the rs744166 polymorphism, the TC genotype (adjusted $\mathrm{OR}=0.60,95 \% \mathrm{CI}=0.39-0.92$, and $P=0.020$ ) and CC genotype (adjusted OR $=0.41$, $95 \% \mathrm{CI}=0.21-0.80$, and $P=0.009$ ) were associated with a decreased risk of gastric cancer compared to the TT genotype. However, rs2293152 did not show any difference in gastric cancer risk between patients and controls in the CG/CC genotype compared to the GG genotype. Besides, the SNP effects were additive to the effects of environmental factors without any interaction between them in the susceptibility to gastric cancer. Collectively, rs744166 polymorphism might be significantly associated with a decreased risk of gastric cancer in a Chinese population. Additionally, polymorphisms in STAT3, along with environmental factors, might be associated with the development of gastric cancer.
\end{abstract}

\section{Introduction}

In recent years, gastric cancer (GC) incidence rates have decreased substantially in most parts of the world. However, GC was still the fourth most common malignancy worldwide, with approximately 989,600 new cases, particularly in Eastern Asian countries, such as China $[1,2]$. It is well known that the development of GC is a multifactorial process that includes both host polymorphisms and environmental factors [3].

Signal transducer and activator of transcription 3 (STAT3) is a key transcription factor of the Janus kinase (JAK)/signal transducer and activator of transcription (STAT) signaling pathway $[4,5]$. The JAK/STAT pathway transmits a wide range of regulatory factors that modulate gene transcription, including cytokines, growth factors, and hormones [6]. In particular, STAT3 can be activated by a variety of ligands that respond to massive signals such as IL-6, TNF- $\alpha$, and VEGF $[4,5,7,8]$. Aberrant expression and constitutive activation of STAT3 are involved in a broad range of human malignancies, including gastric, breast, prostate, and nonsmall cell lung cancers [9-12]. Recent studies have identified STAT3 activation as a key event in regulating cell growth, motility, migration, invasion, angiogenesis, and immune response in GC $[8,9,13-17]$. Furthermore, it was reported that Helicobacter pylori (H. pylori) CagA protein activated the STAT3 signaling pathway in gastric 
cells, providing a potential mechanism by which chronic $H$. pylori infection promotes the development of GC $[18,19]$.

STAT3 is located on chromosomal region $17 \mathrm{q} 21$. To date, single-nucleotide polymorphisms (SNPs) in STAT3 have shown significant associations with cervical cancer, nonsmall cell lung cancer, leukemia, prostate cancer, and hepatocellular cancer [20-24]. Ferguson et al. also found that STAT3 SNPs are significantly associated with susceptibility of Crohn's disease [25]. To our knowledge, no studies have been published to explore the association between STAT3 polymorphisms and GC risk until now.

In this case-control study we aim to evaluate the associations between STAT3 polymorphisms and susceptibility of GC, as well as discussing potential environmental factors.

\section{Materials and Methods}

2.1. Study Subjects. A case-control study was conducted using a Chinese study population of 209 GC patients and 294 controls. All patients, based on pathologic diagnosis, were recruited from the Third Affiliated Hospital of Harbin Medical University. For the frequency-matched controls on age and sex, 154 healthy individuals were recruited from Harbin Center for Disease Control, as well as 140 cancer-free patients who were chosen from the neurology department at the Fourth Affiliated Hospital of Harbin Medical University as controls. All cases and controls had completed a faceto-face questionnaire. A $5 \mathrm{~mL}$ sample of venous blood was collected from each subject following the interview. Cases and controls with incomplete questionnaires, as well as non-GC patients, were excluded from this study. Informed consent was obtained from all subjects and the protocol was approved by the Human Research and Ethics Committee of Harbin Medical University.

2.2. Data Collection. Demographic and habit related data such as age, gender, smoking (including cigarette and pipe) and drinking history, family history of cancer, and frequency of food consumption were collected using a structured questionnaire. We defined smokers as those who smoked more than one cigarette/pipe per day for at least half a year. Likewise, drinkers were defined as those who consumed two or more alcoholic drinks per week for at least half a year. For family history, it referred to first and second degree relatives (parents, grandparents, siblings, and offspring).

2.3. SNP Selection and Genotyping. Genomic DNA was extracted from whole blood using a QIAamp DNA Blood Mini Kit (Qiagen, Germany). DNA purity and concentrations were determined by spectrophotometric measurement of absorbance at 260/280 $\mathrm{nm}$.

STAT3 gene polymorphisms were reviewed based on previously published literature which identified functional effects or associations with disease. Minor allele frequency (MAF) of $\geq 5 \%$ in the Asian population, parameters set by the SNP database of the National Center for Biotechnology Information, were also reviewed. Ultimately, rs2293152 $(\mathrm{G}>\mathrm{C})$ and rs744166 (T>C) polymorphisms were selected.

Polymerase chain reaction restriction fragment length polymorphism (PCR-RFLP) was implemented to determine the genotypes. The PCR primers were designed using Primer Premier 5.0 software. For rs2293152, the primer sequences were $5^{\prime}$-TAGAGGCTTCCTTTTGTTCCG-3' (forward) and $5^{\prime}$-CCAGTTGTCTTTCATCCC-3' (reverse) that generated the 356-bp fragment. For rs744166, the primer sequences were $5^{\prime}$-GAGTACAAACCCTGAACC-3' (forward) and $5^{\prime}$ GACTTGGTGACTGACTGAA-3 ${ }^{\prime}$ (reverse) that generated the 301-bp fragment. Amplification was performed under the following conditions: an initial denaturation for $5 \mathrm{~min}$ at $95^{\circ} \mathrm{C}$, followed by 30 cycles of denaturation at $95^{\circ} \mathrm{C}$ for $30 \mathrm{~s}$, annealing at $56^{\circ} \mathrm{C}$ for $30 \mathrm{~s}$, and extension at $72^{\circ} \mathrm{C}$ for $30 \mathrm{~s}$, followed by a final extension at $72^{\circ} \mathrm{C}$ for $7 \mathrm{~min}$. The amplified fragments of STAT3 rs2293152 and rs744166 were digested by restriction enzymes AciI and AluI (New England BioLabs), respectively, 5 units for $16 \mathrm{~h}$ at $37^{\circ} \mathrm{C}$, followed by electrophoresis on a $2 \%$ agarose gel.

We used the common genotype homozygotes, the rare genotype homozygotes, and the heterozygous of the polymorphisms (rs2293152 and rs744166) for direct sequencing.

2.4. Statistical Analysis. A chi-square $\left(\chi^{2}\right)$ test was used to evaluate Hardy-Weinberg equilibrium (HWE), as well as comparing the genotype and allele frequencies between groups. The associations between genetic polymorphisms and the risk of GC were estimated by computing the odds ratios (ORs) and their 95\% confidence intervals (CIs) from unconditional univariate and multivariate logistic regression analyses. We evaluated the interactions between genetic polymorphisms and environmental factors on the risk of $\mathrm{GC}$ with four types of ORs $\left(\mathrm{OR}_{e}, \mathrm{OR}_{\mathrm{g}}, \mathrm{OR}_{\mathrm{eg}}\right.$, and $\left.\mathrm{OR}_{\mathrm{i}}\right)$. Reference groups consisted of individuals who were not exposed to environmental factors and lacked genetic variants. $\mathrm{OR}_{\mathrm{e}}$ compares the risk of GC based on the level of exposure to environmental factors; $\mathrm{OR}_{\mathrm{g}}$ is the odds ratio for the association between the genetic variant with the risk of $\mathrm{GC} ; \mathrm{OR}_{\text {eg }}$ is the odds ratio for the combined effect of both genetic variants and environmental factors; $\mathrm{OR}_{\mathrm{i}}$ is the odds ratio of interactions of genetic variants and environmental factors. $\left(\mathrm{OR}_{\mathrm{i}}=\mathrm{OR}_{\mathrm{eg}} / \mathrm{OR}_{\mathrm{e}} \mathrm{OR}_{\mathrm{g}}, \mathrm{OR}_{\mathrm{i}}>1\right.$ indicated a positive interaction (synergy); $\mathrm{OR}_{\mathrm{i}}<1$ indicated a negative interaction (antagonism); $\mathrm{OR}_{\mathrm{i}}=1$ indicated that there was no interaction.) A $P$ value of $<0.05$ was considered statistically significant. All tests were two-sided, and all statistical analyses were conducted with SAS 9.1 (SAS Institute, Cary, North Carolina, USA).

\section{Results}

3.1. Characteristics of Study Subjects. This case-control study enrolled 209 GC patients and 294 cancer-free controls. The demographic information on study subjects are shown in Table 1. There were no significant differences between cases 
TABLE 1: Baseline characteristics of study subjects.

\begin{tabular}{|c|c|c|c|}
\hline Characteristics & $\begin{array}{c}\text { Cases } \\
(N=209)\end{array}$ & $\begin{array}{l}\text { Controls } \\
(N=294)\end{array}$ & $P$ value* \\
\hline & No. (\%) & No. (\%) & \\
\hline Age (years) & & & 0.331 \\
\hline$<60$ & $106(50.7)$ & $162(55.1)$ & \\
\hline$\geq 60$ & $103(49.3)$ & $132(44.9)$ & \\
\hline Sex & & & 0.076 \\
\hline Male & $158(75.6)$ & $201(68.4)$ & \\
\hline Female & $51(24.4)$ & 93 (31.6) & \\
\hline \multicolumn{4}{|l|}{$\mathrm{BMI}^{\mathrm{a}}\left(\mathrm{kg} / \mathrm{m}^{2}\right)$} \\
\hline Mean \pm SD & $22.4 \pm 3.8$ & $24.2 \pm 3.3$ & $<0.001$ \\
\hline Smoking status ${ }^{\mathrm{a}}$ & & & $<0.001$ \\
\hline Never & $71(34.1)$ & $154(52.6)$ & \\
\hline Ever & $137(65.9)$ & $139(47.4)$ & \\
\hline Alcohol consumption & & & $<0.001$ \\
\hline Nondrinkers & $85(40.7)$ & $172(58.5)$ & \\
\hline Drinkers & $124(59.3)$ & $122(41.5)$ & \\
\hline Family history of cancer ${ }^{a b}$ & & & $<0.001$ \\
\hline No & $157(77.0)$ & $280(95.9)$ & \\
\hline Yes & $47(23.0)$ & $12(4.1)$ & \\
\hline \multicolumn{4}{|l|}{ TNM stage $\mathrm{e}^{\mathrm{a}}$} \\
\hline I & $14(7.3)$ & & \\
\hline II & $15(7.8)$ & & \\
\hline III & $142(74.0)$ & & \\
\hline IV & $21(10.9)$ & & \\
\hline
\end{tabular}

BMI: body mass index (weight/height ${ }^{2}$ ).

${ }^{*} P$ value from two-sample $t$ tests and $\chi^{2}$ tests.

${ }^{a}$ Missing data: BMI: 7; smoking: 2; family history of cancer: 7; tumor site: 17.

${ }^{b}$ Family history of cancer: gastric, esophageal, liver, colorectal, and other cancers.

and controls in the distributions of age and sex $(P>0.05)$. However, smoking and drinking were overrepresented in the cases compared to the controls $(P<0.001)$, and cases with family history of cancer were more frequent than controls $(P<0.001)$.

3.2. Association between STAT3 Polymorphisms and GC. In our study population, the alleles at rs2293152 and rs744166 loci were in $\operatorname{HWE}(P=0.233$ for $\mathrm{rs} 2293152$ and $P=0.851$ for rs744166). The genotype distributions of these two SNP loci in the cases and controls are presented in Table 2. The distributions of the genotypes for STAT3 rs744166 were 9.1\% (TT), $42.6 \%$ (TC), and $48.3 \%$ (CC) in the cases and $36.4 \%$ (TT), $48.3 \%$ (TC), and $15.3 \%$ (CC) in control subjects ( $P=$ 0.01 ). Additionally, STAT3 rs744166 showed a statistically significant association in further logistic regression analysis. Compared to the TT genotype carriers, the TC genotype (adjusted $\mathrm{OR}=0.60,95 \% \mathrm{CI}=0.39-0.92$, and $P=0.020$ ) and CC genotype (adjusted OR $=0.41,95 \% \mathrm{CI}=0.21-0.80$, and $P=0.009)$ carriers were both less frequent among cases than controls, suggesting a dominant effect of the $\mathrm{C}$ allele. Overall, C carriers (either TC or CC) were associated with a lower GC risk when compared to those carrying the TT genotype (adjusted OR $=0.55,95 \% \mathrm{CI}=0.37-0.83$, and $P=0.004)$. Conversely, the genotype and allele frequencies of locus rs2293152 did not show a significant difference between GC cases and controls.

3.3. Stratified Analysis of STAT3 Polymorphism rs 744166 and GC Risk. We further evaluated the association between genotypes of these two selected SNPs of STAT3 and GC risk by stratified subgroups of age, sex, smoking status, drinking consumption, and family history of cancer, assuming a dominant genetic model (Table 3). Stratified analysis revealed that a decreased risk of GC was associated with the rs744166 TC+CC genotypes among subjects 60 years of age and older (adjusted $\mathrm{OR}=0.49,95 \% \mathrm{CI}=0.26-0.90$, and $P=0.021$ ), but not in subjects less than 60 years old. Additionally, TC+CC genotypes were associated with a decreased risk of GC in male subjects (adjusted OR $=0.54,95 \% \mathrm{CI}=0.32-0.88$, and $P=0.014$ ), whereas the association was not statistically significant in female subjects. When stratified by smoking status, the $\mathrm{C}$ carriers were found to be significantly associated with GC (adjusted OR $=0.54,95 \% \mathrm{CI}=0.32-0.93$, and $P=$ 0.026) compared to the TT genotype carriers in smokers. Likewise, drinkers with $\mathrm{C}$ carriers showed a significantly decreased risk of GC (adjusted OR $=0.38,95 \% \mathrm{CI}=0.21-0.70$, and $P=0.002$ ), compared to those carrying the TT genotype. Stratification by family history of cancer revealed a significant association of TC+CC genotypes and the risk of GC (adjusted $\mathrm{OR}=0.55,95 \% \mathrm{CI}=0.36-0.85$, and $P=0.007)$ in subjects without family history of cancer, whereas the association was not statistically significant in subjects with family history of cancer. Conversely, we observed no statistical significance between the rs 2293152 gene polymorphism and the risk of GC (data not show).

3.4. Combined Effects of STAT3 Polymorphisms on GC Risk. Table 4 shows analyses of interaction and combination effects between genotypes and environmental exposures for the risk of GC. We found that these two SNPs effects were additive to the effects of environmental factors without any interaction between them in the susceptibility to GC.

For rs744166, significant combination effects between high intake of chicken ( $\geq$ average 2 times/week) and fresh fruit ( $\geq$ average 2 times/week) and the TC+CC genotypes were observed (chicken: $\mathrm{OR}_{\mathrm{eg}}=0.25$ and $95 \% \mathrm{CI}=$ $0.13-0.48$; fresh fruit: $\mathrm{OR}_{\mathrm{eg}}=0.34$ and $95 \% \mathrm{CI}=0.19$ 0.61 ). Consumption of seafood ( $\geq$ average 2 times/week) and using a refrigerator to store food were associated with a decreased risk of GC in subjects with a $\mathrm{C}$ allele or TT genotype. On the contrary, long-term irregular eating habits were associated with an increased risk of GC. Additionally, no statistically significant interactions or combination effects were observed between consumption of pork, preference 
TABLE 2: Genotype and allele frequencies of STAT3 polymorphisms and their associations with GC risk.

\begin{tabular}{|c|c|c|c|c|c|c|}
\hline Genotypes & $\begin{array}{c}\text { Cases }(N=209) \\
\text { Number }(\%)\end{array}$ & $\begin{array}{c}\text { Controls }(N=294) \\
\text { Number }(\%)\end{array}$ & OR (95\% CI) & $P$ value & $\mathrm{OR}_{\mathrm{adj}}(95 \% \mathrm{CI})^{\mathrm{a}}$ & $P$ value \\
\hline \multicolumn{7}{|l|}{ rs2293152 } \\
\hline GG & $56(26.8)$ & $74(25.2)$ & 1.00 & & 1.00 & \\
\hline CG & $94(45.0)$ & $157(53.4)$ & $0.79(0.51-1.22)$ & 0.287 & $0.87(0.54-1.41)$ & 0.540 \\
\hline $\mathrm{CC}$ & $59(28.2)$ & $63(21.4)$ & $1.24(0.75-2.03)$ & 0.400 & $1.40(0.81-2.42)$ & 0.330 \\
\hline CG/CC & & & $0.92(0.61-1.38)$ & 0.681 & $1.03(0.66-1.62)$ & 0.893 \\
\hline \multicolumn{7}{|l|}{ rs744166 } \\
\hline $\mathrm{TT}$ & $101(48.3)$ & $107(36.4)$ & 1.00 & & 1.00 & \\
\hline TC & $89(42.6)$ & $142(48.3)$ & $0.66(0.45-0.97)$ & 0.035 & $0.60(0.39-0.92)$ & 0.020 \\
\hline $\mathrm{CC}$ & $19(9.1)$ & $45(15.3)$ & $0.45(0.25-0.82)$ & 0.009 & $0.41(0.21-0.80)$ & 0.009 \\
\hline $\mathrm{TC} / \mathrm{CC}$ & & & $0.61(0.43-0.88)$ & 0.008 & $0.55(0.37-0.83)$ & 0.004 \\
\hline
\end{tabular}

OR: odds ratio; CI: confidence interval.

${ }^{a}$ Adjusted for age, sex, smoking, drinking, BMI, and family history of cancer.

for hot food, and the rs744166 polymorphism on GC risk. Moreover, the combination effects of rs2293152 and environmental factors are almost identical to rs744166 (data shown in Table 4).

\section{Discussion}

GC is an inflammation-related disease that induces massive cytokine release, including IL-1, IL-6, IL-12, and TNF- $\alpha$ [2628]. STAT3, which transmits a wide range of cytokines, was first identified in 1994 as an IL-6-activated acute-phase response factor (APRF) [29]. It is frequently reported to be overexpressed in various cancers [30] and has, therefore, been recognized as an oncogene. Several STAT3 SNPs were reported to be significantly associated with cervical cancer, nonsmall cell lung cancer, metastatic renal cell carcinoma, prostate cancer, and hepatocellular cancer. These include rs2293152, rs744166, rs4769793, rs4796793, and rs7211777 [4, 20, 23, 24]. Moreover, rs744166 was also reported to have a significant association with Crohn's disease [25, 31]. Based on previous studies, our case-control study detected the effect of STAT3 rs2293152 and rs744166 gene polymorphisms on GC. In our study, we found that subjects with the minor C allele of rs744166 had a decreased risk of developing GC compared to the common genotype in a Chinese population. However, we did not find any significant association between the rs2293152 gene polymorphism and risk of GC.

Recently, increasing evidence shows that polymorphisms of the STAT3 gene are associated with various diseases [21, 3133]. In a large German cohort study, a significant association was observed between the minor allele of STAT3 rs744166 and Crohn's disease $(\mathrm{OR}=0.83,95 \% \mathrm{CI}=0.688-0.998$, and $P=$ 0.04) [31]. Similarly, Danoy et al. identified STAT3 rs744166 as a susceptible loci of ankylosing spondylitis $(\mathrm{OR}=0.84$, $95 \% \mathrm{CI}=0.77-0.91$, and $P=2.6 \times 10^{-5}$ ) in a population of white European ancestry from the UK, USA, Canada, and Australia [34]. Moreover, Jiang et al. reported that the minor allele of rs744166 significantly decreased the risk of nonsmall cell lung cancer in a Chinese population [20]. These findings on the rs744166 C allele and the decreased risk of GC in the present study are consistent with reported results. However, the results of our study on rs2293152 show variation from previous research. A Chinese study involving 1,021 hepatocellular carcinoma patients and 1,012 healthy controls found that rs2293152 (GG versus CC) was significantly associated with risk of hepatocellular carcinoma [24]. Yet, other researches on familial breast cancer and nonsmall cell lung cancer did not show associations of rs2293152 [20,33] and neither did our study. These differences could potentially be the result of varying environmental backgrounds, ethnic groups, and sample sizes.

Furthermore, our present study revealed a decreased effect with the C allele of rs744166 in subgroups of males, smokers, and drinkers. Stratified analysis suggested that the association between the $\mathrm{C}$ allele of rs744166 and the risk of GC was more profound in males than females. A previous study reported that noncardia cancer was more common in males than females by a ratio of approximately $2: 1$, and gastric cardia cancer had a ratio of nearly 4.1:1 in a Chinese population [35]. Our data suggests that STAT3 polymorphism may play an important role in males with GC. Smoking, which generates reactive oxygen species production and induces DNA adducts that may lead to mutations and thus the initiation of carcinogenesis, has been confirmed to be an independent risk factor of GC $[36,37]$. Meanwhile, for drinking, previous reports suggested that alcohol consumption is one of the risk factors of GC as well $[38,39]$. In our present study, it is also suggested that the influence of the C allele of rs744166 on GC was more critical in subgroups of smokers and drinkers. The underlying mechanism involved in the association between STAT3 and smoking and drinking is not clear. It is likely that smoking 
TABLE 3: Stratified analyses of rs744166 genotypes between GC cases and controls.

\begin{tabular}{|c|c|c|c|}
\hline \multirow{2}{*}{ Variables } & \multicolumn{2}{|r|}{ rs744166 } & \multirow{2}{*}{$P$ value } \\
\hline & TT & $\mathrm{TC}+\mathrm{CC}$ & \\
\hline \multicolumn{4}{|l|}{ Age (years) } \\
\hline \multicolumn{4}{|l|}{$<60$} \\
\hline Cases/controls & $48 / 61$ & $58 / 101$ & \\
\hline $\mathrm{OR}_{\mathrm{adj}}(95 \% \mathrm{CI})^{\mathrm{a}}$ & 1.00 & $0.64(0.37-1.13)$ & 0.127 \\
\hline \multicolumn{4}{|l|}{$\geq 60$} \\
\hline Cases/controls & $53 / 46$ & $50 / 86$ & \\
\hline $\mathrm{OR}_{\mathrm{adj}}(95 \% \mathrm{CI})^{\mathrm{a}}$ & 1.00 & $0.49(0.26-0.90)$ & 0.021 \\
\hline \multicolumn{4}{|l|}{ Sex } \\
\hline \multicolumn{4}{|l|}{ Male } \\
\hline Cases/controls & $77 / 73$ & $81 / 128$ & \\
\hline $\mathrm{OR}_{\mathrm{adj}}(95 \% \mathrm{CI})^{\mathrm{a}}$ & 1.00 & $0.54(0.32-0.88)$ & 0.014 \\
\hline \multicolumn{4}{|l|}{ Female } \\
\hline Cases/controls & $24 / 34$ & $27 / 59$ & \\
\hline $\mathrm{OR}_{\mathrm{adj}}(95 \% \mathrm{CI})^{\mathrm{a}}$ & 1.00 & $0.63(0.28-1.40)$ & 0.254 \\
\hline \multicolumn{4}{|l|}{ Smoking status ${ }^{\mathrm{b}}$} \\
\hline \multicolumn{4}{|l|}{ Never } \\
\hline Cases/controls & $32 / 58$ & $39 / 96$ & \\
\hline $\mathrm{OR}_{\mathrm{adj}}(95 \% \mathrm{CI})^{\mathrm{a}}$ & 1.00 & $0.56(0.29-1.07)$ & 0.081 \\
\hline \multicolumn{4}{|l|}{ Ever } \\
\hline Cases/controls & $68 / 48$ & $69 / 91$ & \\
\hline $\mathrm{OR}_{\mathrm{adj}}(95 \% \mathrm{CI})^{\mathrm{a}}$ & 1.00 & $0.54(0.32-0.93)$ & 0.026 \\
\hline \multicolumn{4}{|l|}{ Alcohol consumption } \\
\hline \multicolumn{4}{|l|}{ Nondrinkers } \\
\hline Cases/controls & $39 / 69$ & $46 / 103$ & \\
\hline $\mathrm{OR}_{\mathrm{adj}}(95 \% \mathrm{CI})^{\mathrm{a}}$ & 1.00 & $0.73(0.40-1.31)$ & 0.288 \\
\hline \multicolumn{4}{|l|}{ Drinkers } \\
\hline Cases/controls & $62 / 38$ & $62 / 84$ & \\
\hline $\mathrm{OR}_{\mathrm{adj}}(95 \% \mathrm{CI})^{\mathrm{a}}$ & 1.00 & $0.38(0.21-0.70)$ & 0.002 \\
\hline \multicolumn{4}{|c|}{ Family history of cancer ${ }^{b c}$} \\
\hline \multicolumn{4}{|l|}{ No } \\
\hline Cases/controls & $76 / 102$ & $81 / 178$ & \\
\hline $\mathrm{OR}_{\mathrm{adj}}(95 \% \mathrm{CI})^{\mathrm{a}}$ & 1.00 & $0.55(0.36-0.85)$ & 0.007 \\
\hline \multicolumn{4}{|l|}{ Yes } \\
\hline Cases/controls & $22 / 4$ & $25 / 8$ & \\
\hline $\mathrm{OR}_{\mathrm{adj}}(95 \% \mathrm{CI})^{\mathrm{a}}$ & 1.00 & $0.50(0.11-2.28)$ & 0.371 \\
\hline
\end{tabular}

OR: odds ratio; CI: confidence interval.

${ }^{a}$ Adjusted for age, sex, smoking, drinking, BMI, and family history of cancer.

${ }^{\mathrm{b}}$ Missing data: smoking: 2; family history of cancer: 7 .

${ }^{c}$ Family history of cancer: gastric, esophageal, liver, colorectal, and other cancers.

and drinking might significantly induce STAT3 expression, and it is possible that the C allele of rs744166 leads to a higher basal expression level of STAT3 under unnormal circumstances. Therefore, the subjects carrying the $\mathrm{C}$ allele of rs744166 might not have decreased GC risk under normal conditions but might have a reduced risk when exposed to tobacco and alcohol that might induce STAT3 expression in response.

Interestingly, we found that the influence of $\mathrm{C}$ allele of rs744166 disappeared in the subjects with family history, although it existed in all study subjects. Positive family history was reported as a strong and consistent risk factor for GC due to the combination result of a number of alleles $[40,41]$. Therefore, the decreased risk effect of rs744166 in subjects with family history may be overwhelmed by the role of familial aggregation, which may partly contribute to the results we observed. Small sample size in the subgroups may also account for the variation seen between studies. Therefore, further studies should be conducted with a larger sample size.

Single SNPs in low-penetrance genes are unlikely to significantly affect susceptibility to cancer, but combination effects may exist $[42,43]$. In our study, significant combination effects were observed with environmental factors. Fruits are high in antioxidants, phytosterols, and other substances that may inhibit carcinogenesis by free-radical quenching or by blocking the formation of $\mathrm{N}$-nitroso compounds. Epidemiologic studies have found that fruits are associated with a decreased risk of GC [44]. In this study, we found a significant combination effect between fresh fruit consumption and the $\mathrm{C}$ allele of rs744166 in decreasing the risk of GC. Meanwhile, lifestyle choices such as longterm irregular eating habits and the methods of storing food may also be important factors for GC in this Chinese population. To our knowledge, this is the first study to be concerned with the interaction between the STAT3 gene polymorphisms and environmental factors for the risk of GC; further studies are needed to confirm this phenomena and a mechanistic explanation for our findings needs to be explored.

Some limitations in our study need to be addressed. First, the relatively small sample size may reduce the statistical power in stratified analysis. The second limitation was an inevitable recall bias during data collection in regard to environmental factors, although measures were taken to minimize this bias. Third, we adopted a frequency questionnaire to collect information about dietary intake that did not take into consideration the amounts of food being consumed. Therefore, the questionnaire had limited power to detect precise gene-dietary interactions. Finally, H. pylori is an independent risk factor for GC that we did not explore due to ethical considerations. These related issues will need to be addressed in our future studies.

In conclusion, our data provided the first evidence that the C allele of STAT3 rs744166 is associated with decreased GC risk in a Chinese population. The minor C allele of rs744166 may correlate with STAT3 expression and thus exert a protective effect on GC development. Additional large-scale, well-designed studies that include environmental factors are required to further validate the role of STAT3 gene polymorphisms in GC risk. 


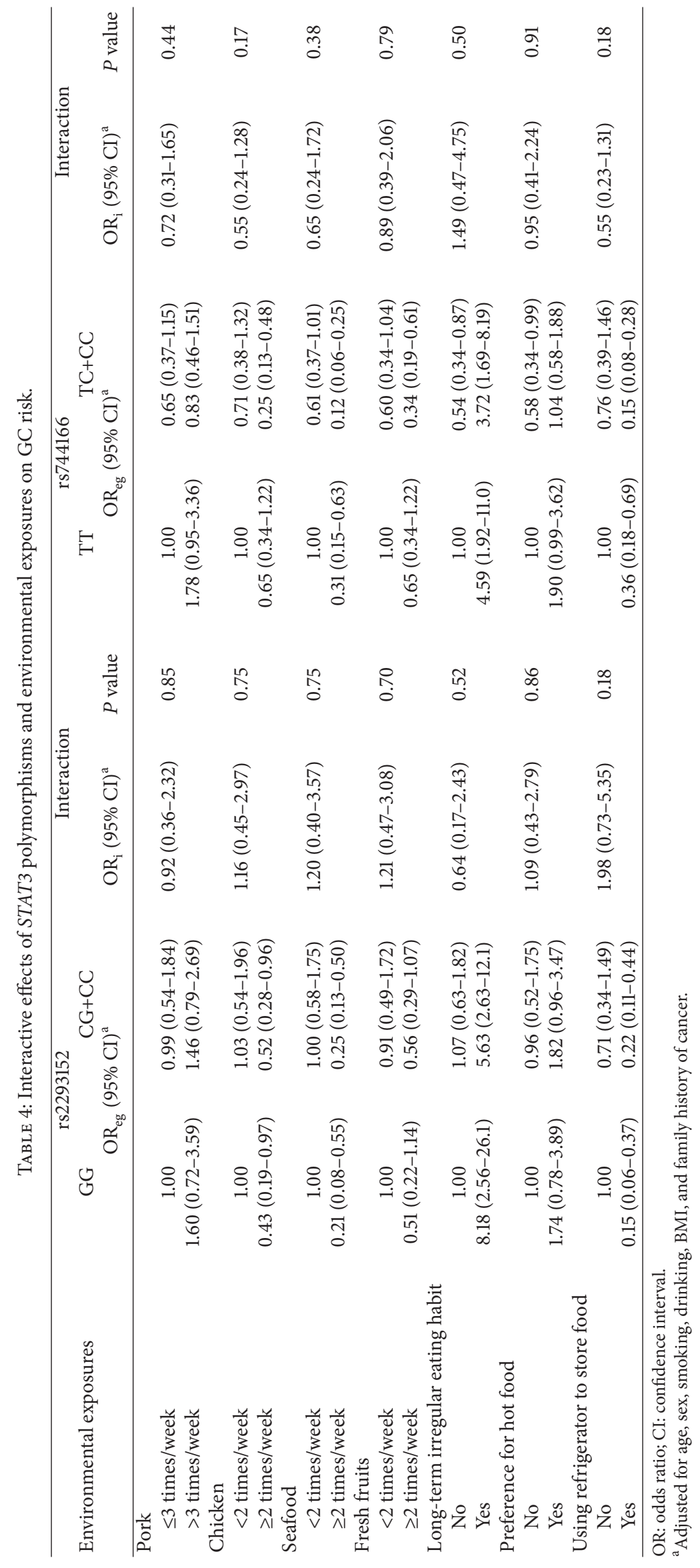




\section{Conflict of Interests}

The authors declare that they have no conflict of interests.

\section{Authors' Contribution}

Kexin Yuan and Huimin Liu contributed equally to this work.

\section{Acknowledgments}

The authors are especially grateful to all of the individuals who made this research. This study was supported by the National Natural Science Foundation of China (20092011, Grant no. 30800939) and the Returned Overseas Scholars Foundation in Heilongjiang (2013-2015, Grant no. LC2012C38).

\section{References}

[1] A. Jemal, F. Bray, M. M. Center, J. Ferlay, E. Ward, and D. Forman, "Global cancer statistics," A Cancer Journal for Clinicians, vol. 61, no. 2, pp. 69-90, 2011.

[2] P. Bertuccio, L. Chatenoud, F. Levi et al., "Recent patterns in gastric cancer: a global overview," International Journal of Cancer, vol. 125, no. 3, pp. 666-673, 2009.

[3] L. E. Wroblewski, R. M. Peek Jr., and K. T. Wilson, "Helicobacter pylori and gastric cancer: factors that modulate disease risk," Clinical Microbiology Reviews, vol. 23, no. 4, pp. 713-739, 2010.

[4] N. Ito, M. Eto, E. Nakamura et al., "STAT3 polymorphism predicts interferon-alfa response in patients with metastatic renal cell carcinoma," Journal of Clinical Oncology, vol. 25, no. 19, pp. 2785-2791, 2007.

[5] J. E. Darnell Jr., "STATs and gene regulation," Science, vol. 277, no. 5332, pp. 1630-1635, 1997.

[6] D. E. Levy and J. E. Darnell Jr., "STATs: transcriptional control and biological impact," Nature Reviews Molecular Cell Biology, vol. 3, no. 9, pp. 651-662, 2002.

[7] A. Sekikawa, H. Fukui, S. Fujii et al., "REG I $\alpha$ protein mediates an anti-apoptotic effect of STAT3 signaling in gastric cancer cells," Carcinogenesis, vol. 29, no. 1, pp. 76-83, 2008.

[8] J. Chen, J. Wang, L. Lin et al., "Inhibition of STAT3 signaling pathway by nitidine chloride suppressed the angiogenesis and growth of human gastric cancer," Molecular Cancer Therapeutics, vol. 11, no. 2, pp. 277-287, 2012.

[9] W. Okamoto, I. Okamoto, T. Arao, K. Yanagihara, K. Nishio, and K. Nakagawa, "Differential roles of STAT3 depending on the mechanism of STAT3 activation in gastric cancer cells," British Journal of Cancer, vol. 105, no. 3, pp. 407-412, 2011.

[10] Z. Ni, W. Lou, S. O. Lee et al., "Selective activation of members of the signal transducers and activators of transcription family in prostate carcinoma," The Journal of Urology, vol. 167, no. 4, pp. 1859-1862, 2002.

[11] T. Zhou, L. Chao, G. Rong, C. Wang, R. Ma, and X. Wang, "Down-regulation of GRIM-19 is associated with STAT3 overexpression in breast carcinomas," Human Pathology, vol. 44, no. 9, pp. 1773-1779, 2013.

[12] S. M. Kim, O. J. Kwon, Y. K. Hong et al., "Activation of IL-6R/JAK1/STAT3 signaling induces De Novo resistance to irreversible EGFR inhibitors in non-small cell lung cancer with
T790M resistance mutation," Molecular Cancer Therapeutics, vol. 11, no. 10, pp. 2254-2264, 2012.

[13] Z. Wei, X. Jiang, H. Qiao et al., "STAT3 interacts with Skp2/p27/p21 pathway to regulate the motility and invasion of gastric cancer cells," Cellular Signalling, vol. 25, no. 4, pp. 931938, 2013.

[14] J. Yoon, S. J. Cho, Y. S. Ko et al., "A synergistic interaction between transcription factors nuclear factor- $\kappa \mathrm{B}$ and signal transducers and activators of transcription 3 promotes gastric cancer cell migration and invasion," BMC Gastroenterology, vol. 13 , p. 29, 2013.

[15] L. A. Denson, "Adding fuel to the fire: STAT3 priming of gastric tumorigenesis," Gastroenterology, vol. 131, no. 4, pp. 1342-1344, 2006.

[16] N. Kanda, H. Seno, Y. Konda et al., "STAT3 is constitutively activated and supports cell survival in association with survivin expression in gastric cancer cells," Oncogene, vol. 23, no. 28, pp. 4921-4929, 2004.

[17] A. S. Giraud, T. R. Menheniott, and L. M. Judd, "Targeting STAT3 in gastric cancer," Expert Opinion on Therapeutic Targets, vol. 16, no. 9, pp. 889-901, 2012.

[18] D. M. Bronte-Tinkew, M. Terebiznik, A. Franco et al., "Helicobacter pylori cytotoxin-associated gene a activates the signal transducer and activator of transcription 3 pathway in vitro and in vivo," Cancer Research, vol. 69, no. 2, pp. 632-639, 2009.

[19] O. Lee, J. H. Kim, Y. J. Choi et al., "Helicobacter pylori CagA phosphorylation status determines the gp130-activated SHP2/ERK and JAK/STAT signal transduction pathways in gastric epithelial cells," The Journal of Biological Chemistry, vol. 285, no. 21, pp. 16042-16050, 2010.

[20] B. Jiang, Z. Z. Zhu, F. Liu et al., "STAT3 gene polymorphisms and susceptibility to non-small cell lung cancer," Genetics and Molecular Research, vol. 10, no. 3, pp. 1856-1865, 2011.

[21] K. Wang, B. Zhou, J. Zhang et al., "Association of signal transducer and activator of transcription 3 gene polymorphisms with cervical cancer in Chinese women," DNA and Cell Biology, vol. 30, no. 11, pp. 931-936, 2011.

[22] Y. Zhong, J. Wu, B. Chen et al., "Investigation and analysis of single nucleotide polymorphisms in Janus kinase/signal transducer and activator of transcription genes with leukemia," Leukemia \& Lymphoma, vol. 53, no. 6, pp. 1216-1221, 2012.

[23] E. M. Kwon, C. A. Salinas, S. Kolb et al., "Genetic polymorphisms in inflammation pathway genes and prostate cancer risk," Cancer Epidemiology Biomarkers and Prevention, vol. 20, no. 5, pp. 923-933, 2011.

[24] J. Xie, Y. Zhang, Q. Zhang et al., "Interaction of signal transducer and activator of transcription 3 polymorphisms with hepatitis $B$ virus mutations in hepatocellular carcinoma," Hepatology, vol. 57, no. 6, pp. 2369-2377, 2013.

[25] L. R. Ferguson, D. Y. Han, A. G. Fraser et al., "Genetic factors in chronic inflammation: single nucleotide polymorphisms in the STAT-JAK pathway, susceptibility to DNA damage and Crohn's disease in a New Zealand population," Mutation Research, Fundamental and Molecular Mechanisms of Mutagenesis, vol. 690, no. 1-2, pp. 108-115, 2010.

[26] S. Odenbreit, S. Linder, B. Gebert-Vogl, G. Rieder, A. P. Moran, and R. Haas, "Interleukin-6 induction by Helicobacter pylori in human macrophages is dependent on phagocytosis," Helicobacter, vol. 11, no. 3, pp. 196-207, 2006. 
[27] N. Hida, T. Shimoyama Jr., P. Neville et al., "Increased expression of IL-10 and IL-12 (p40) mRNA in Helicobacter pylori infected gastric mucosa: relation to bacterial cag status and peptic ulceration," Journal of Clinical Pathology, vol. 52, no. 9, pp. 658-664, 1999.

[28] U. E. H. Mai, G. I. Perez-Perez, L. M. Wahl, S. M. Wahl, M. J. Blaser, and P. D. Smith, "Soluble surface proteins from Helicobacter pylori activate monocytes/macrophages by lipopolysaccharide-independent mechanism," The Journal of Clinical Investigation, vol. 87, no. 3, pp. 894-900, 1991.

[29] C. Lutticken, U. M. Wegenka, J. Yuan et al., "Association of transcription factor APRF and protein kinase Jakl with the interleukin-6 signal transducer gp130," Science, vol. 263, no. 5143, pp. 89-92, 1994.

[30] H. Yu and R. Jove, "The stats of cancer-new molecular targets come of age," Nature Reviews Cancer, vol. 4, no. 2, pp. 97-105, 2004.

[31] M. Prager, J. Büttner, V. Haas et al., "The JAK2 variant rs10758669 in Crohn's disease: altering the intestinal barrier as one mechanism of action," International Journal of Colorectal Disease, vol. 27, no. 5, pp. 565-573, 2011.

[32] K. Hu, S. Hou, Z. Jiang, A. Kijlstra, and P. Yang, "JAK2 and STAT3 polymorphisms in a Han Chinese population with Behçet's disease," Investigative ophthalmology \& visual science, vol. 53, no. 1, pp. 538-541, 2012.

[33] A. Vaclavicek, J. L. Bermejo, R. K. Schmutzler et al., "Polymorphisms in the Janus kinase $2(J A K) /$ signal transducer and activator of transcription (STAT) genes: putative association of the STAT gene region with familial breast cancer," EndocrineRelated Cancer, vol. 14, no. 2, pp. 267-277, 2007.

[34] P. Danoy, K. Pryce, J. Hadler et al., "Association of variants at 1q32 and STAT3 with ankylosing spondylitis suggests genetic overlap with Crohn's disease," PLoS Genetics, vol. 6, no. 12, 2010.

[35] J. R. Kelley and J. M. Duggan, "Gastric cancer epidemiology and risk factors," Journal of Clinical Epidemiology, vol. 56, no. 1, pp. $1-9,2003$.

[36] B. Secretan, K. Straif, R. Baan et al., "A review of human carcinogens-part E: tobacco, areca nut, alcohol, coal smoke, and salted fish," The lancet oncology, vol. 10, no. 11, pp. 1033-1034, 2009.

[37] S. S. Hecht, "Tobacco smoke carcinogens and breast cancer," Environmental and Molecular Mutagenesis, vol. 39, no. 2-3, pp. 119-126, 2002.

[38] I. Tramacere, E. Negri, C. Pelucchi et al., "A meta-analysis on alcohol drinking and gastric cancer risk," Annals of Oncology, vol. 23, no. 1, Article ID mdr135, pp. 28-36, 2012.

[39] Y. Li, H. Yang, and J. Cao, "Association between alcohol consumption and cancers in the chinese population-a systematic review and meta-analysis," PLoS ONE, vol. 6, no. 4, Article ID e18776, 2011.

[40] M. A. Han, M. G. Oh, I. J. Choi et al., "Association of family history with cancer recurrence and survival in patients with gastric cancer," Journal of Clinical Oncology, vol. 30, no. 7, pp. 701-708, 2012.

[41] M. Yaghoobi, R. Bijarchi, and S. A. Narod, "Family history and the risk of gastric cancer," British Journal of Cancer, vol. 102, no. 2, pp. 237-242, 2010.

[42] C.-J. Weng, C.-W. Lin, T.-T. Chung, C.-M. Tsai, M.-K. Chen, and S.-F. Yang, "Impact of uPA system gene polymorphisms on the susceptibility of environmental factors to carcinogenesis and the development of clinicopathology of oral cancer," Annals of Surgical Oncology, vol. 18, no. 3, pp. 805-812, 2011.

[43] P. Vineis, "Diet, genetic susceptibility and carcinogenesis," Public Health Nutrition, vol. 4, no. 2, pp. 485-491, 2001.

[44] S. A. N. Silvera, S. T. Mayne, H. A. Risch et al., "Principal component analysis of dietary and lifestyle patterns in relation to risk of subtypes of esophageal and gastric cancer," Annals of Epidemiology, vol. 21, no. 7, pp. 543-550, 2011. 


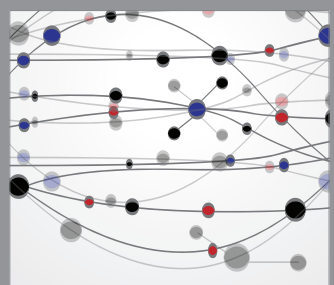

The Scientific World Journal
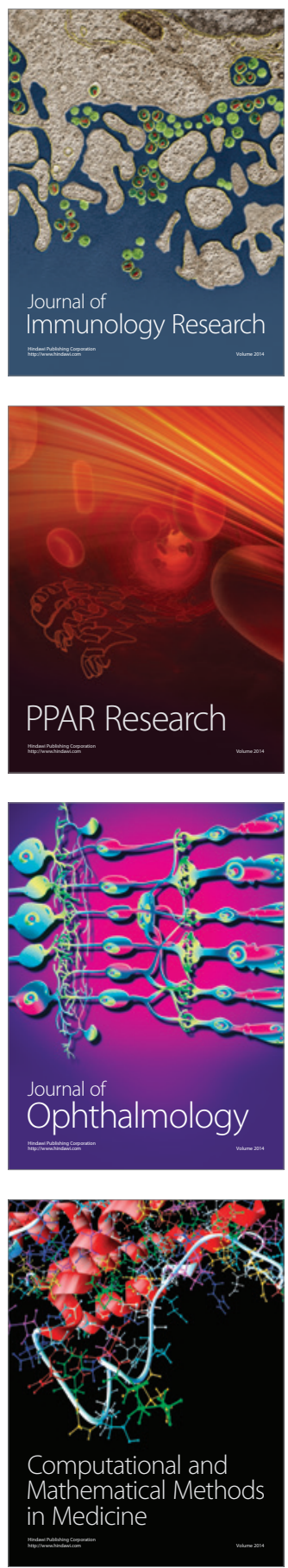

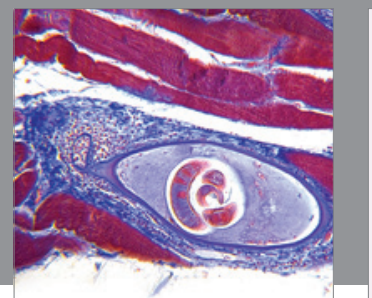

Gastroenterology

Research and Practice
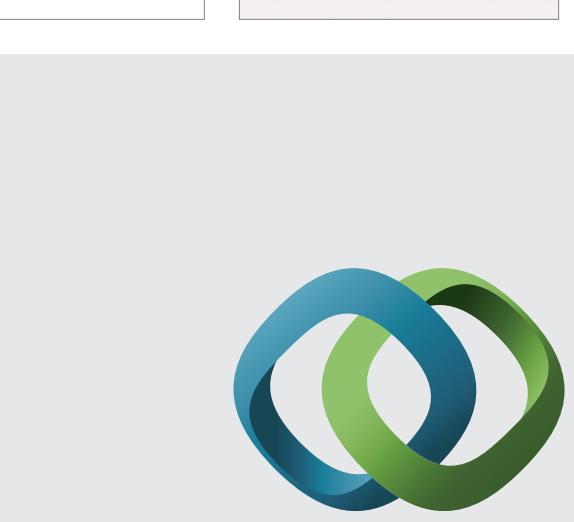

\section{Hindawi}

Submit your manuscripts at

http://www.hindawi.com
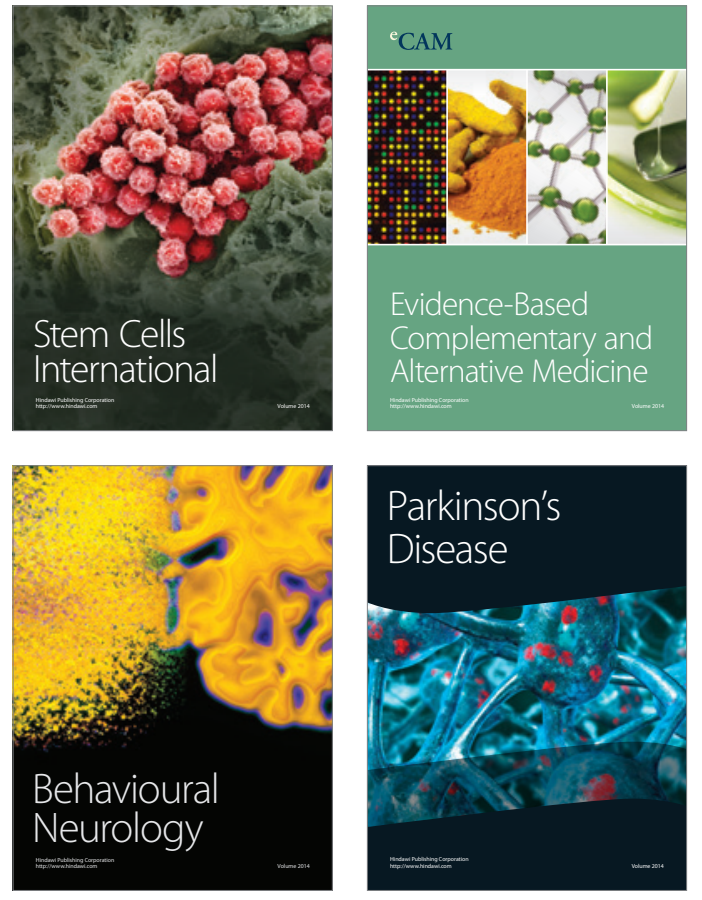
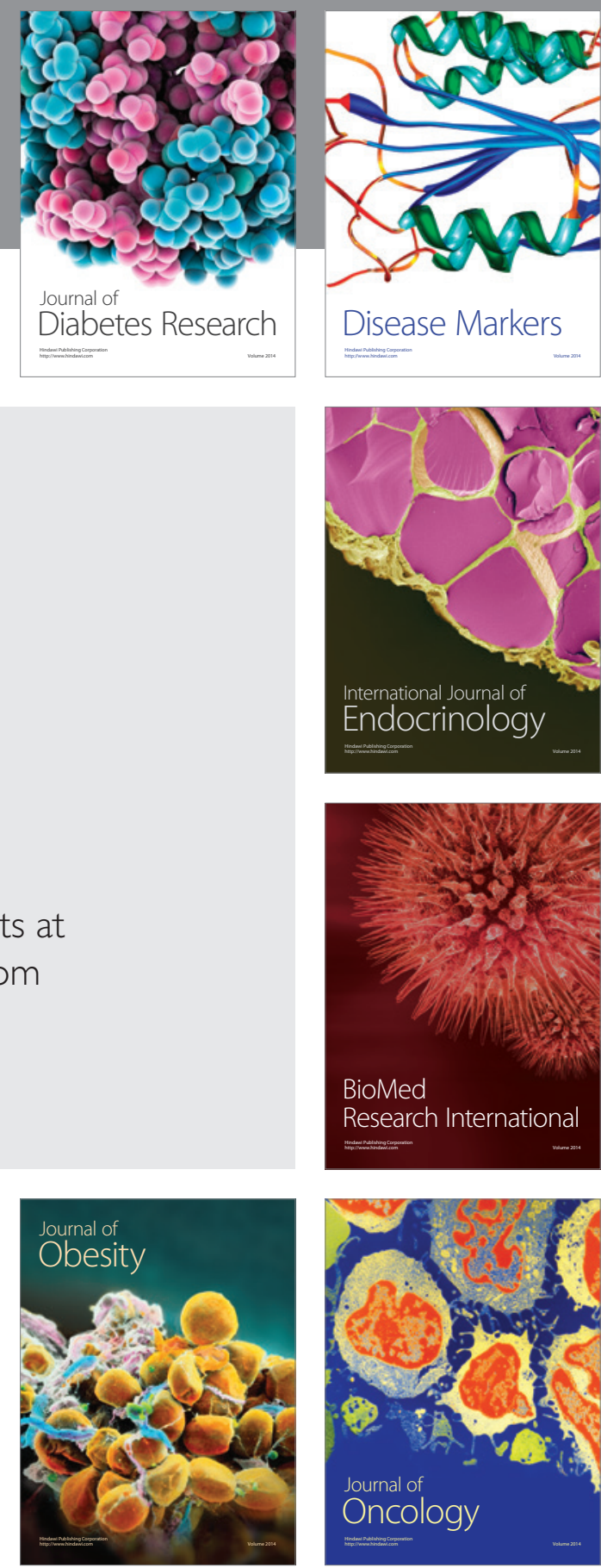

Disease Markers
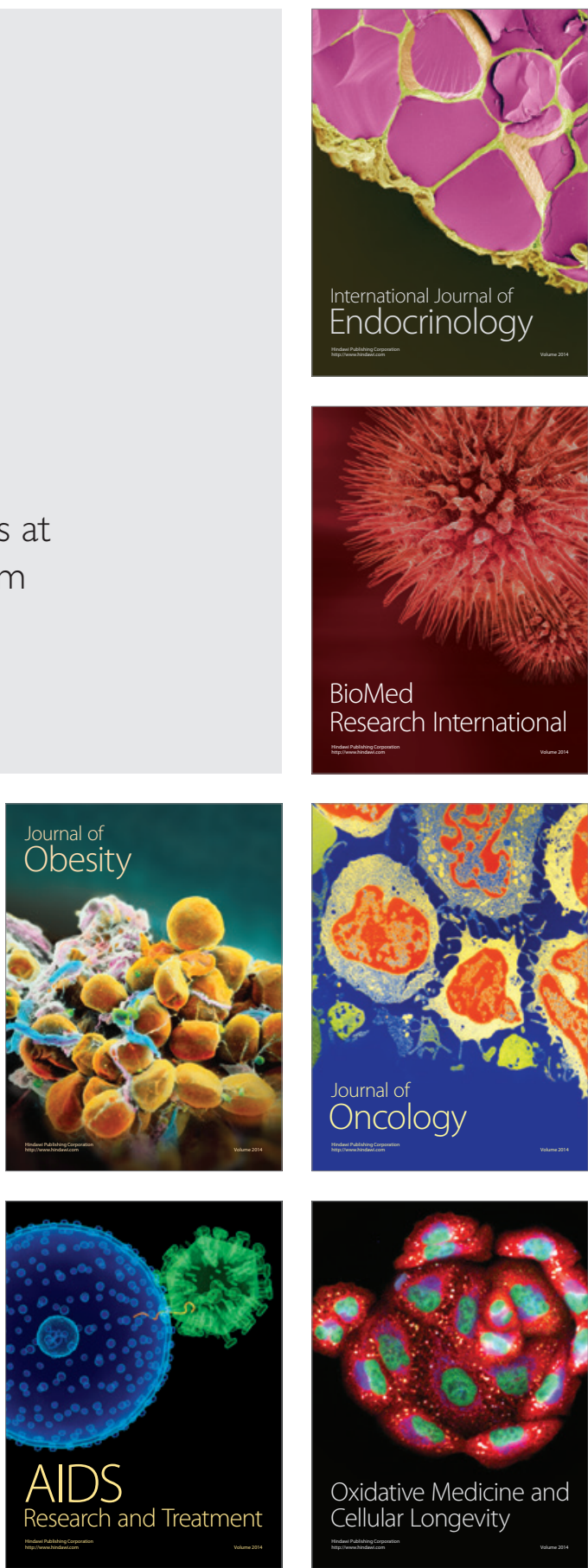